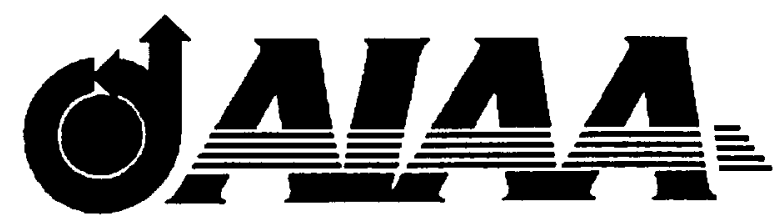

NASA-CR-201457

\title{
AIAA 95-1846
}

Three-Dimensional Aerodynamic Analysis of a Subsonic Transport High-Lift Configuration and Comparisons with Wind-Tunnel Test Results

D. Christian Edge and John N. Perkins North Carolina State University Raleigh, NC 27695-7910

\section{3th AIAA Applied Aerodynamics Conference June 19-22, 1995/San Diego, CA}




\title{
THREE-DIMENSIONAL AERODYNAMIC ANALYSIS OF A SUBSONIC TRANSPORT HIGH-LIFT CONFIGURATION AND COMPARISONS WITH WIND-TUNNEL TEST RESULTS
}

\author{
D. Christian Edge * and John N. Perkins ${ }^{\dagger}$ \\ North Carolina State University \\ Raleigh, NC 27695-7910
}

\begin{abstract}
The sizing and efficiency of an aircraft is largely determined by the performance of its high-lift system. Subsonic civil transports most often use deployable multi-element airfoils to achieve the maximum-lift requirements for landing, as well as the high lift-to-drag ratios for take-off. However, these systems produce very complex flow fields which are not fully understood by the scientific community. In order to compete in today's market place, aircraft manufacturers will have to design better high-lift systems. Therefore, a more thorough understanding of the flows associated with these systems is desired.

Flight and wind-tunnel experiments have been conducted on NASA Langley's B737-100 research aircraft to obtain detailed full-scale flow measurements on a multi-element high-lift system at various flight conditions. As part of this effort, computational aerodynamic tools are being used to provide preliminary flow-field information for instrumentation development, and to provide additional insight during the data analysis and interpretation process. The purpose of this paper is to demonstrate the ability and usefulness of a three-dimensional low-order potentialflow solver, PMARC, by comparing computational results with data obtained from $1 / 8$ scale wind-tunnel tests. Overall, correlation of experimental and computational data reveals that the panel method is able to predict reasonably well the pressures of the aircraft's multi-element wing at several spanwise stations. PMARC's versatility and usefulness is also demonstrated by accurately predicting inviscid threedimensional flow features for several intricate geometrical regions.
\end{abstract}

\footnotetext{
- Research Assistant, Mechanical and Aerospace Engineering, Student Member AIAA.

'Professor, Mechanical and Aerospace Engineering, Associate Fellow AIAA.
}

Copyright (c) 1995 by the American Institute of Aeronautics and Astronautics, Inc. All rights reserved.

\section{Nomenclature}

$C_{L_{\text {mex }}}$ maximum lift coefficient

$C_{P} \quad$ pressure coefficient

$L / D \quad$ lift-to-drag ratio

$R n \quad$ Reynolds number

$\alpha \quad$ aircraft angle of attack, (deg)

$\eta \quad$ non-dimensional span location, $\mathrm{y} /(\mathrm{b} / 2)$

$\Delta s \quad$ step size used in streamline traces

Abbreviations

B737-100

CFD

CPU

PMARC

TSRV

Boeing 737-100

Computational Fluid Dynamics

Central Processing Unit

Panel Method Ames Research Center

Transport Systems Research Vehicle

\section{Introduction}

The primary objective in high-lift system design is to safely achieve airfield requirements without deteriorating cruise performance. This usually necessitates high maximum-lift, $C_{L_{\text {max }}}$, capabilities for landing as well as high lift-to-drag, $L / D$, ratios for take-off. Modern jet transports incorporate complex multielement systems to achieve such conditions, and the current competitive market is requiring aircraft companies to design simpler, more efficient high-lift systems. However, the complex flow fields associated with these systems make the design problem a difficult task. ${ }^{1}$

Traditionally, wind-tunnel tests were used as the primary means for evaluating multi-element highlift flows; however, testing itself is limited by costs, and the low Reynolds number experimental data has limited applicability to flight Reynolds number conditions. ${ }^{2}$ Only recently, with the evolution of computer hardware capabilities, have CFD tools become a viable option for assessing the viscous and inviscid effects present in high-lift flows. ${ }^{3,4,5}$ Current limitations in computer resources and flow modeling techniques still restrict most viscous computational solutions to only two dimensions; however, the need still exists for an accurate and cost efficient means for 
predicting inviscid three-dimensional effects. ${ }^{6,} 7$ Twodimensional codes will always be limited by the accuracy of the models which they use to account for sweep, taper, and finite-wing effects when analyzing a 3-D wing. Furthermore, 2-D codes will always lack the ability to accurately and solely predict sectional flows dominated by $3-D$ effects, such as regions near the nacelles, wing tips, or fuselage. Thus, it is essential when examining a high-lift system to isolate three-dimensional effects so that their prominence in the flow physics can be accessed.

Until a full viscous 3-D solution becomes practical, alternate methods must combine 2-D viscous solutions with 3-D inviscid calculations to provide the best analysis of a multi-element high-lift system. The NASA Langley high-lift flight-research team is currently using this type of approach to evaluate the B737-100 research aircraft. ${ }^{8}$ The purpose of this computational effort is to: (1) provide preliminary flowfield information during the instrumentation development and installation process; (2) provide additional information during the data analysis and interpretation process; and (3) determine the usefulness as well as the limitations of the available CFD codes in the study of high-lift aerodynamics for complex configurations. Three-dimensional potential-flow solutions about the aircraft in its landing-approach configuration have been calculated using PMARC (Panel Method Ames Research Center).

\section{Computational Analyses}

Potential-flow simulations of transport aircraft with high-lift devices have been recognized as being an essential step in the evolution of a rational analytical design capability and have also served as a foundation for viscous/vortex simulations of these configurations. ${ }^{9}$ These codes are often advantageous over other flow solvers because of their ability to model complex configurations without getting involved with flow-field grid generations. They are also favored for many applications due to their relative economy. Several studies have proven that potentialflow panel methods are capable of successfully analyzing transport aircraft configurations. ${ }^{10,11}$ When used for high-lift purposes, panel methods can be beneficial by identifying regions of predominantly 2-D flows, detecting areas of highly interactive 3-D flows, and determining regions which are dominated by vortex flows. In the past, higher-order methods were usually applied when the velocity near the body was continuous, such as the gaps of multi-element airfoils. However, constant strength singularity elements have shown their potential to give good results in these regions when a sufficient number of panels are used and when proper panel spacing is applied. ${ }^{12,} 13,14$

\section{Code Presentation}

PMARC (Version 12.19), was developed by Ashby et al. ${ }^{15}$ at the NASA Ames Research Center as an incompressible potential-flow panel method capable of numerically predicting inviscid flow fields around complex three-dimensional geometries. The part of the flow potential which represents the body is modeled by constant-strength source and doublet distributions over each panel, thus the code is referred to as a low-order method. The internal Dirichlet boundary condition is prescribed to each panel on the surface so that the velocity potential for each control point can be calculated. This way, the sources are determined explicitly based on external normal velocities, and the doublet strengths are determined using an integral equation which prescribes the condition on the inner potential. The code also has the option of employing the Neumann boundary condition which works by directly specifying the zero normal velocity component at each control point on the patch. PMARC does include an optional boundary-layer routine to account for viscous effects; however, this analysis, based on the momentum-integral equation, was deemed to be not accurate enough for the present problem. Therefore, only inviscid solutions are presented.

\section{Surface Grid Generation}

Creating the computational surface mesh can sometimes be one of the most difficult and timeconsuming procedures when applying a panel method to a complex configuration. Without a robust geometry preprocessor, surface modeling can become tedious and cumbersome. GEOMAIR was chosen to create and manipulate the surface grid of the B737100 high-lift configuration. This code was developed at North Carolina State University and provides a simple user interface for creating complex threedimensional geometries for several different aerodynamic panel codes. ${ }^{16}$ It was designed to perform various geometrical transformations, including the rotation and scaling of individual surface components. Each modeled geometry consists of a number of curved regions called "patches", which are divided up jnto quadrilateral "panels". Each patch consists of at least two "sections" which are a series of points linked together defining the surface curvature.

The surface mesh of the Boeing 737-100 was created using coordinates obtained from the Boeing Commercial Airplane Group. Figure 1 shows the surface grid of the starboard wing without the engine nacelle. This approach configuration (also referred to as the $40^{\circ}$ flap configuration) consists of nearly 5,100 
panels while representing only half of the airplane. This final number of panels, with proper panel spacing and alignment, was able to accurately capture the complexity of the geometry while at the same time provide detailed pressure distributions.

When the engine nacelle was added to the model, an additional 1,100 panels were appended to the surface grid (Figure 2). Each finalized mesh was the result of sensitivity studies which were implemented while developing the model so that the least number of panels could be used to capture the flow behavior of interest. These studies were conducted in both the chordwise and spanwise directions for every patch. The ultimate panel density was determined when negligible changes in the solution occurred as the number of panels was increased.

Several geometry changes had to be made to the computational model in order to minimize numerical complications. One such change was the smoothing of the cove regions on the main element and mid flap. These areas are allocated for storing the retracted trailing-edge flap system during cruise conditions. Since potential-flow methods often have diffculties in differentiating the doublet strengths around sharp surface discontinuities, the coves were reshaped to simulate the displacement of the separation bubbles which exist in these regions during viscous conditions. The faired coves were developed during previous 2-D studies on the same wing at the $\eta=0.58$ station. Viscous 2-D analyses revealed negligible differences in pressures between the two cove shapes. ${ }^{17,18}$

Another notable difference between the computational model and the actual aircraft is the removal of the slat "lip" which abuts the lower aft side of the leading-edge device. Favorably, the 1/8 scale windtunnel model did not include the "lip" on its geometry, thus resulting errors were eliminated when only compared to tunnel data. A cross-sectional view of the computational geometry at the $\eta=0.58$ semispan station is shown in Figure 3.

\section{Wake Modeling}

Lifting effects are modeled in PMARC using vortex wakes. Wakes can be shed from known separation lines on the surface geometry in order to fix the rear stagnation point and the circulation around the body. These wakes must be attached to the trailing edge of any surface which uses the Kutta condition as a boundary condition. The Kutta condition requires the velocity at the rear stagnation point to be finite, and is essential for determining the doublet strengths on the first row of panels of the trailing vortex sheet.

Previous studies on the same high-lift configuration concentrated on examining several different wake models using PMARC. ${ }^{12,19}$ It was concluded from these studies that a "streamline method" was the best wake model when examining this high-lift configuration. This particular wake model uses the results of off-body streamline traces to form a defined wake geometry. This systematic approach was accepted with the understanding that the wakes are generally aligned with the local velocity field (or offbody streamlines). ${ }^{13} \mathrm{~A}$ similar technique would be to form the wake lattice from detailed flow visualizations; however, these types of results were not available for the current study.

When creating the "streamline wake", a flat rigid wake lattice was initially used while tracing streamlines from the trailing edge of each lifting element. In order to achieve adequate roll-up definition for the $40^{\circ}$ flap configuration, a step size $(\Delta s)$ of 5 inches was used. Off-body traces were computed to a finite distance downstream equal to approximately one span length. A short FORTRAN algorithm was developed to convert the computed streamlines into the proper format for PMARC wakes. Solutions were then recomputed using the new wake geometries.

Figure 4 shows the off-body streamline traces for the B737-100 at a typical approach angle $\left(\alpha=8.0^{\circ}\right)$. The presence of roll-up is distinctly evident near the edges of all lifting surfaces. Notice that the strongest region of vortical flow appears to generate off the tip of the outboard trailing-edge flap system. This flow phenomenon correlates well with visual observations commonly seen during flight and wind-tunnel tests. A greater acceptance of the streamline wake method may be established by observing a cross-sectional cut of the off-body traces. Figure 5 shows a planar view at the $\eta=0.58$ semispan station and reveals very realistic wake shapes for the five-element wing.

\section{Description of the Research Aircraft}

The NASA-Langley Transport Systems Research Vehicle (TSRV) was the prototype aircraft used in the development of the Boeing 737-100 series. While still maintaining its original exterior shape, the research vehicle has been significantly modified while at NASA to accommodate a variety of different fight tests. ${ }^{20}$ In order to achieve short-field take-offs and landings, the-aircraft incorporates a multi-element high-lift system. The leading-edge high-lift devices consist of an inboard Kruger flap and three outboard slats. The trailing-edge assemblage is comprised of two sets of triple-slotted Fowler flaps separated by the engine nacelle. Therefore, a typical wing section consists of five elements: slat (or Kruger flap), main wing, fore flap, mid flap, and aft flap. Detailed flow measurements have been obtained at one nominal spanwise station 
$(\eta=0.58)$ from previous flight tests. ${ }^{8,} 21$

\section{Wind-Tunnel Experiments}

Wind-tunnel tests were conducted on a $1 / 8$ scale model of the TSRV in the Langley $14 \times 22$ foot subsonic tunnel. A photograph of the model while installed in the tunnel is shown in Figure 6. The objective of the $14 \times 22$ tests was to obtain surface pressure measurements for understanding flow physics, verifying CFD codes, and comparing with full-scale flight results.

Nearly 650 flush pressure taps were distributed at the seven different spanwise stations illustrated in Figure 7. These taps were placed on both the upper and lower surfaces of every applicable high-lift element at each of the spanwise locations. The windtunnel tests were conducted within dynamic pressures of $10 \mathrm{psf}$ and $40 \mathrm{psf}$. As a result, Mach numbers varied between 0.08 to 0.17 , and Reynolds numbers ranged from 0.54 to 1.07 million. The test matrix included angles-of-attack between $-4^{\circ}$ and $14^{\circ}$ for the $15^{\circ}, 30^{\circ}$, and $40^{\circ}$ flap configurations.

\section{Discussion of Results}

During typical approach maneuvers, the TSRV extends all leading-edge devices to their proper positions, and deploys its trailing-edge flap system to a $40^{\circ}$ setting. This high-lift configuration is used to produce maximum lift for landing and was the only configuration investigated. Since total force coefficients were not compared in this study, the tail surfaces were removed from the computational geometry. Values for the computational chordwise pressure coefficients were extracted by linearly interpolating between the two nearest control point columns on each element. A specified convergence criteria of 0.0005 was used by the matrix solver, and was normally attained within 190 iterations. This convergence tolerance is defined as the percent change in the solution vector elements between successive iterations. The element in the solution vector with the largest change is used to determine if the solution is converged.

As mentioned earlier, chordwise pressure distributions were measured at seven different spanwise stations on the $1 / 8$ scale wind-tunnel model. Five of these stations incorporate all five high-lift elements. Presently, flight-test data is limited to only one spanwise station $(\eta=0.58)$; therefore, it will only be presented in comparisons at that section. However, extensive comparisons between PMARC and flight data have been made in the past and are summarized in References [12] and [19].
Comparison of Flight, Wind-Tunnel and PMARC Pressures

Figure 8 compares flight, wind-tunnel, and PMARC pressures at the $\eta=0.58$ wing section. Despite Reynolds number differences, flight and tunnel data agree reasonably well at the given angle-ofattack; however, the magnitude of the pressure peaks on the leading edge of the main wing are noticeably different. This can be attributed to the absence of the slat "lip" on the wind-tunnel model which effectively reduces the circulation around the entire slat element. As a result, the loading on the aft portion of the slat decreases and the magnitude of the pressure peak on the main element increases. It is evident that the PMARC model, also lacking the slat "lip", was able to capture this inviscid effect by closely predicting the location and magnitude of the first pressure peak.

Neither PMARC nor wind-tunnel tests could replicate the double pressure peaks on the leading edge as measured in flight. The second peak is attributed to the local flow acceleration caused by a notch in the upper surface where the slat nests during cruise conditions. PMARC solutions correspond reasonably well with she rest of the main wing's experimental pressures except for the last quarter of the upper surface where the boundary-layer influence is greatest. Evident by the close agreement between the last few points on the wing's lower surface, the modified cove was effective in simulating the separation bubble in this region. The high experimental $C_{P}$ values near the aft portion of the wing indicate that the flow reattaches at the trailing edge. This correlates well with the fully-attached solutions obtained from the code.

Similar to experimental data, computational results indicated that the pressures at the trailing edge of each flap element, except for the aft flap, did not completely recover due to the influence of the downstream element. As expected, the code overpredicts the upper-surface flap pressures; however, the uppersurface trends were legitimately matched and lowersurface pressures were nearly identical. These results were anticipated due to the absence of prominent viscous/compressible effects which are present in these regions during flap deployment (contiuent boundary layers, wake/boundary layer interacticns, etc.).

\section{Comparison of Pressures over Slat 3}

Comparisons between wind-tunnel and PMARC pressures at the $\eta=0.54$ wing section are presented in Figure 9. It should be noted that this spanwise station incorporates Slat 3 , which has a smaller deflection than Slats 1-2 (see Figure 1). As a result, the 
effective angle-of-attack on the slat element is greater. Consequently, the slat loading is larger for this section than stations which incorporate the other two slats. PMARC does well at predicting the increased pressure loading and sharp peak on the slat element.

Another notable flow feature which PMARC is able to predict at the $\eta=0.54$ section is the small "notch" that occurs on the pressure peak of the main wing. This notch is attributed to the large overhang that exists between the slat and main element. As a result, the circulation around the slat is able to suppress the pressure peak on the main wing until the trailing edge of the forward element is encountered.

\section{Kruger Flap Modeling}

A comparison was made between computational and experimental pressures at the $\eta=0.23$ semispan station. As observed in Figure 10, this particular section incorporates the deployed Kruger flap as its leading-edge device. The Kruger flap itself is a very thin surface which abuts the lower front side of the main wing. Since the surface has a "near zero" thickness with no available geometrical representation of the adjacent separation bubble, the lifting element cannot be treated as an enclosed body in PMARC. Thus, the Dirichlet boundary condition is no longer valid for this patch since the internal potential cannot be assumed constant. The Neumann boundary condition, which prescribes a condition on the surface itself, had to be applied to the Kruger patch.

Figure 10 compares PMARC pressures with those obtained from the wind-tunnel. Again, very good correlation is established between the experimental and computational data. By prescribing the Neumann boundary condition on the Kruger flap, the panel method is able to accurately predict the pressure peak on the main element. To demonstrate the effectiveness of applying the Neumann condition to the Kruger flap, solutions were also computed using the Dirichlet condition and are presented in the same figure. The results of this change were highly inaccurate pressures over the forward portion of the main element. No pressure data was available for the Kruger flap; therefore, the accuracy of the computed pressures for this surface cannot be verified. The success achieved on leading-edge devices at other spanwise sections would indicate that PMARC's Kruger pressures are reasonable.

\section{Effects of the Engine Nacelle}

As stated earlier, an additional 1,100 panels were appended to the geometry when the nacelle and pylon were added to the model. No boundary conditions were placed on the inlet or exit of the engine, thus power effects were not calculated. Previous studies revealed that the engine nacelle addition produces minimal pressure differences at the $\eta=0.58$ spanwise station. ${ }^{12,19}$ It was concluded, however, that the addition would have a more prominent effect on wing sections closer to the pylon.

Figure 11 shows the effects that the engine nacelle addition has at the $\eta=0.40$ semispan station. Indeed, the better representation of the true geometry improves the predictions on the slat surface and on the forward portion of the main wing. However, the solution degrades towards the aft portion of the main wing and over each of the trailing-edge elements. The small gaps that exist between the flap tips and nacelle create erroneous suction pressures on surrounding panels. Establishing a good model for such regions is very difficult when using panel methods. Since the potential flow method does not account for viscous effects, the narrow gaps cause velocities within the local vicinity to increase to unrealistic values, which in reality are reduced by viscous friction. Past studies reveal that the best approach when applying a low-order method to such regions, is to simply leave the wing tips open. ${ }^{22}$ However, this technique had already been incorporated into the TSRV geometry with regards to other numerical considerations. Thus, the effects of the nacelle addition at the selected semispan station can be best summarized as improving the accuracy of slat pressures, with the consequential degradation of flap predictions.

\section{Effects of Panel Spacing}

Comparisons at the $\eta=0.71$ wing section are presented in Figure 12 and are included to demonstrate the importance of panel spacing when modeling a multi-element system. Spacing was optimized for the $\eta=0.58$ section so that the most accurate solution could be obtained with the least number of panels. Unfortunately, the gaps and overlaps of the TSRV's outboard flap and slat elements vary according to span. As a result, the numerical accuracy degrades away from the $\eta=0.58$ section. This consequence is clearly evident in Figure 12 by the sharp pressure spikes on the leading edge of each flap element. A solution to this $3-D$ modeling problem would be to greatly increase the density of panels near the multi-element gaps.

An attempt was made to resolve the pressure spikes at the $\eta=0.71$ section. This was done by doubling the number of chordwise panels on each flap element. Although small reductions in discrepancies could be achieved, the amount of CPU time required to accomplish this task overshadowed any net benefits. The TSRV high-lift configuration without en- 
gine nacelles $(\approx 5100$ panels) required 2046 seconds of CPU time on Langley's Cray Y-MP supercomputer. The same configuration with doubled chordwise flap panels ( $\approx 6500$ panels), required 2719 seconds of CPU time. Furthermore, there was no true advantage of resolving the pressure spikes in the flap regions since large amounts of error already existed as a result of the absence of viscous and compressible effects.

\section{Sources of Error}

Several primary factors can account for the discrepancies between experimental and computational results in this study. Most important is the absence of viscous effects in the potential-flow solutions. Although PMARC has the ability to compensate for 3-D finite wing-effects, the inviscid code largely overpredicts regions dominated by boundary layers and flow separation. Likewise, the absence of compressible effects is acknowledged as a source of error especially between the gaps of high-lift elements.

The geometry of the B737-100 high-lift system under flight loads is presently not defined enough for the measured data to serve as a benchmark. This has become a major interest for researchers and is one of the primary topics currently being investigated in the B737-100 high-lift flight research program at NASA Langley. Additional geometrical error was introduced as a result of the alterations made to the computational model. These include the removal of the slat "lip" and the smoothing of the cove shapes.

\section{Concluding Remarks}

A multi-phased flight program is underway at the NASA Langley Research Center to obtain detailed characteristics of multi-element high-lift flows. In support of this research, wind-tunnel tests have been conducted on a $1 / 8$ scale model of the NASA Langley Transport Systems Research Vehicle. Several CFD tools are being used in the program to assist with instrumentation development and planning, and to provide additional information for the data analysis and interpretation process.

PMARC, a low-order panel method, has been used to provide an inviscid three-dimensional analysis of the Boeing 737-100 in its high-lift configuration. Overall, correlations between experimental and computational data reveal that the panel method is able to predict reasonably well the pressure distributions of a multi-element wing. Comparisons made at various spanwise stations demonstrate the code's versatility and usefulness by accurately predicting inviscid 3-D flow features in complex geometrical regions.

\section{Acknowledgments}

This work was supported in part by NASA Grant NCC1-154.

\section{References}

[1] Smith, A.M.O., "High-Lift Aerodynamics," Journal of Aircraft, Vol. 12, No. 6, June 1975, pp. 501-530.

[2] Valarezo, W.O., Dominik, C.J., McGhee, R.J. Goodman, W.L., and Paschal, K.B., "MultiElement Airfoil Optimization for Maximum Lift at High Reynolds Numbers," AIAA Paper 913332, September 1991.

[3] Garner, P.L., Meredith, P.T., and Stoner, R.C., "Areas for Future CFD Development as Illustrated by Transport Aircraft Applications," AIAA Paper 91-1527-CP, June 1991.

[4] Meredith, P.T., "Viscous Phenomena Affecting High-Lift Systems and Suggestions for Future CFD Development," AGARD-CP-415, September 1993 .

[5] Tinoco, E.N., "CFD Applications to Complex Configurations: A Survey," Progress In Astronautics and Aeronautics: Applied Computational Aerodynamics, Vol. 125, AIAA, Washington D.C., 1990, pp. 559-615.

[6] Brune, G.W., McMaters, J.H., "Computational Aerodynamics Applied to High-Lift Systems," Progress In Astronautics and Aeronautics: Applied Computational Aerodynamics, Vol. 125, AIAA, Washington D.C., 1990, pp. 389-433.

[7] Rogers, S.E., "Progress in High-Lift Aerodynamic Calculations," AIAA Paper 93-0194, January 1993.

[8] Yip, L.P., and et al., "Overview of the NASA B737-100 High-Lift Flight Research Program," ICAS Paper 94-1.1.2, September, 1994.

[9] Dillner, B., May, F.W., and McMasters, J.H., "A Method for Predicting Low-Speed Aerodynamic Characteristics of Transport Aircraft," Journal of Aircraft, Vol. 21, March 1984, pp. $168-174$.

[10] Tinoco, E.N., Ball, D.N., and Rice, F.A.,"PAN AIR Analysis of a Transport High-Lift Configuration," Journal of Aircraft, Vol. 24, March 1987, pp. 181-187. 
[11] Murillo, L.E., and McMasters, J.H., "A Method for Predicting Low-Speed Aerodynamic Characteristics of Transport Aircraft," AIAA Paper 83-1845, July 1983.

[12] Edge, D.C., "Three Dimensional Computational Aerodynamic Analysis of a Transport High-Lift Configuration," Master's Thesis, North Carolina State University, July 1994.

[13] Roggero, F., and Larguier, R., "Aerodynamic Calculation of Complex Three-Dimensional Configurations," Journal of Aircraft, Vol. 30, No. 5, September 1993, pp. 561-570.

[14] Dodbele, S.S., "Three Dimensional Aerodynamic Analysis of a High-Lift Transport Configuration," AIAA Paper 93-3536, August 1993.

[15] Ashby, D.L., Dudley, M.R., Iguchi, S.K., Browne, L., and Katz, J., "Potential Flow Theory and Operation Guide for the Panel Code PMARC," NASA TM-102851, January 1991.

[16] Rhodes, G.S., "GEOMAIR: Geometry PrepProcessor User's Manual," Mechanical and Aerospace Engineering Department, North Carolina State University, May 1989.

[17] Hardin, J.D., Potter, R.C., van Dam, C.P., and Yip, L.P., "Two-dimensional Computational Analysis of a Transport High-Lift System and Comparison with Flight-Test Results," AIAA Paper 93-3533, August 1993.

[18] Potter, R.C., "Viscous-Flow Analysis of a Subsonic Transport Aircraft High-Lift System and Correlation with Flight Data," Master's Thesis, University of California at Davis, June 1994.

[19] Edge, D.C., and Perkins, J.N., "ThreeDimensional Aerodynamic Analysis of a Subsonic Transport High-Lift Configuration Using PMARC," AIAA Paper 95-0039, January 1995.

[20] White, J.J., "Advanced Transport Operating Systems Program," SAE Paper 901969, October, 1990.

[21] Yip, L.P., Vijgen, P.M.H.W., Hardin, J.D., and van Dam, C.P., "In-Flight Pressure Distributions and Skin Friction Measurements on a Subsonic Transport High-Lift Wing Section," AGARD-CP-415, October 1992.

[22] Katz, J., and Plotkin, A., Low-Speed Aerodynamics: From Wing Theory to Panel Methods, McGraw-Hill, Inc., 1991. 


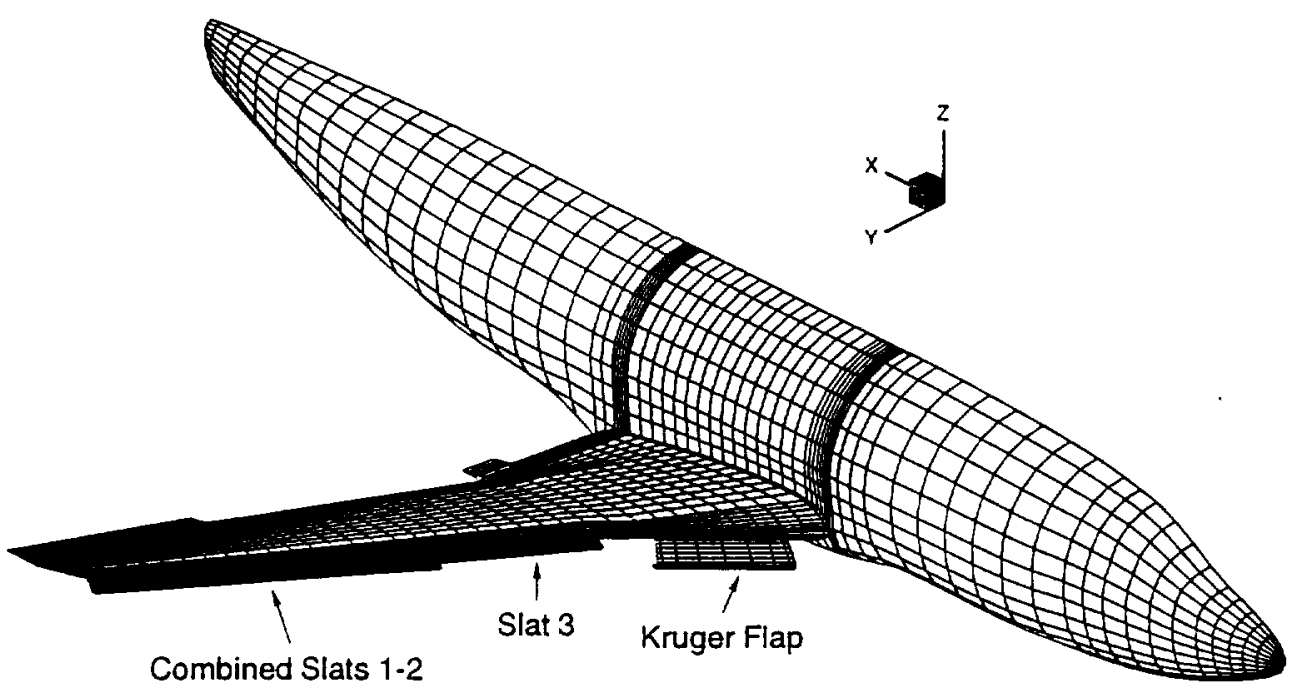

Figure 1. Front View of Paneled Approach Configuration

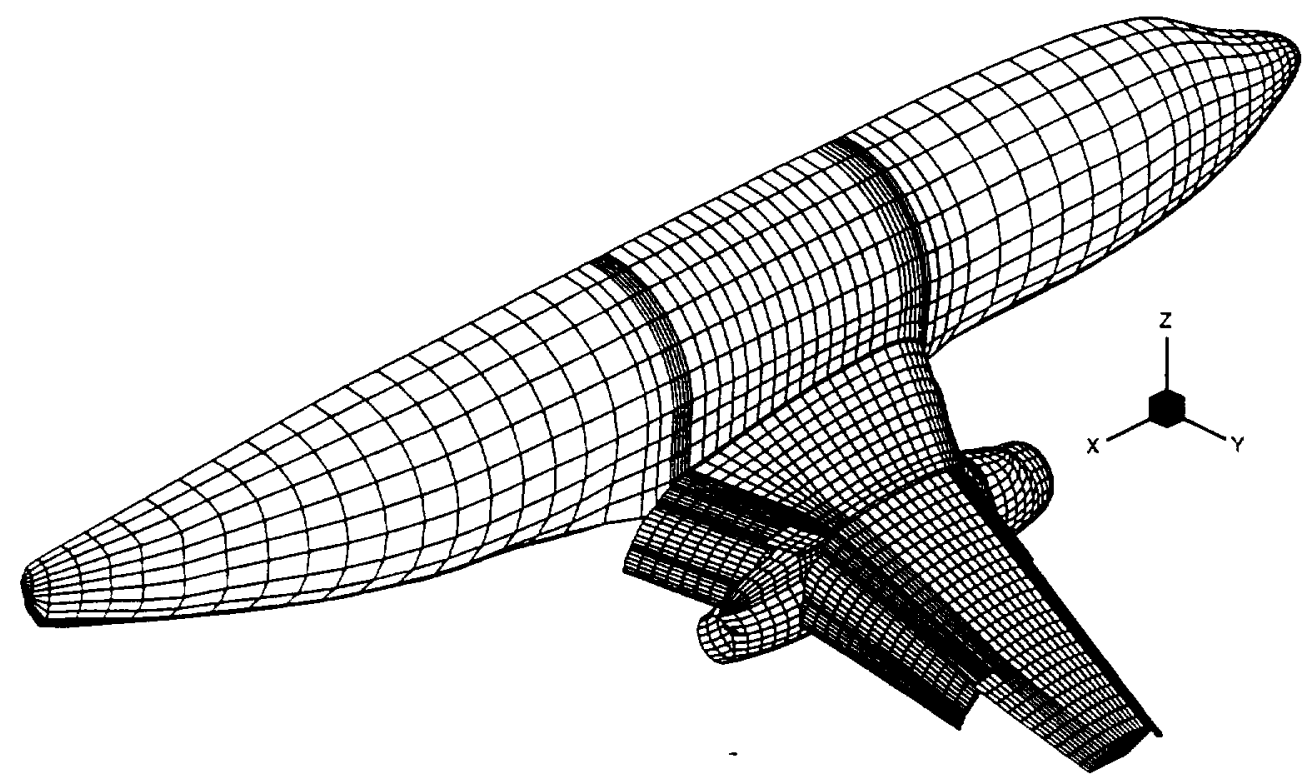

Figure 2. Surface Grid of Approach Configuration with Engine Nacelle 


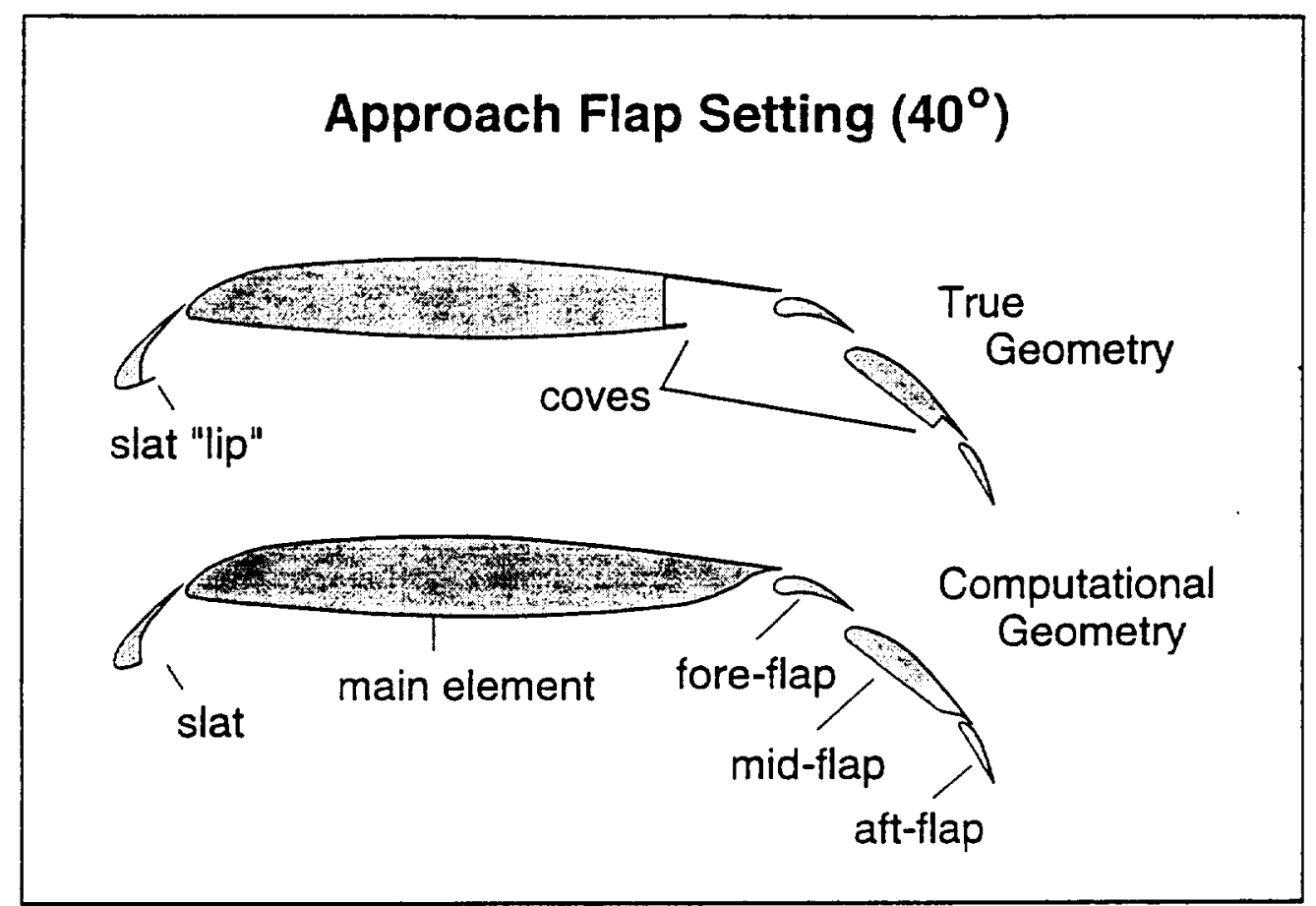

Figure 3. Comparison of True and Computational Geometries

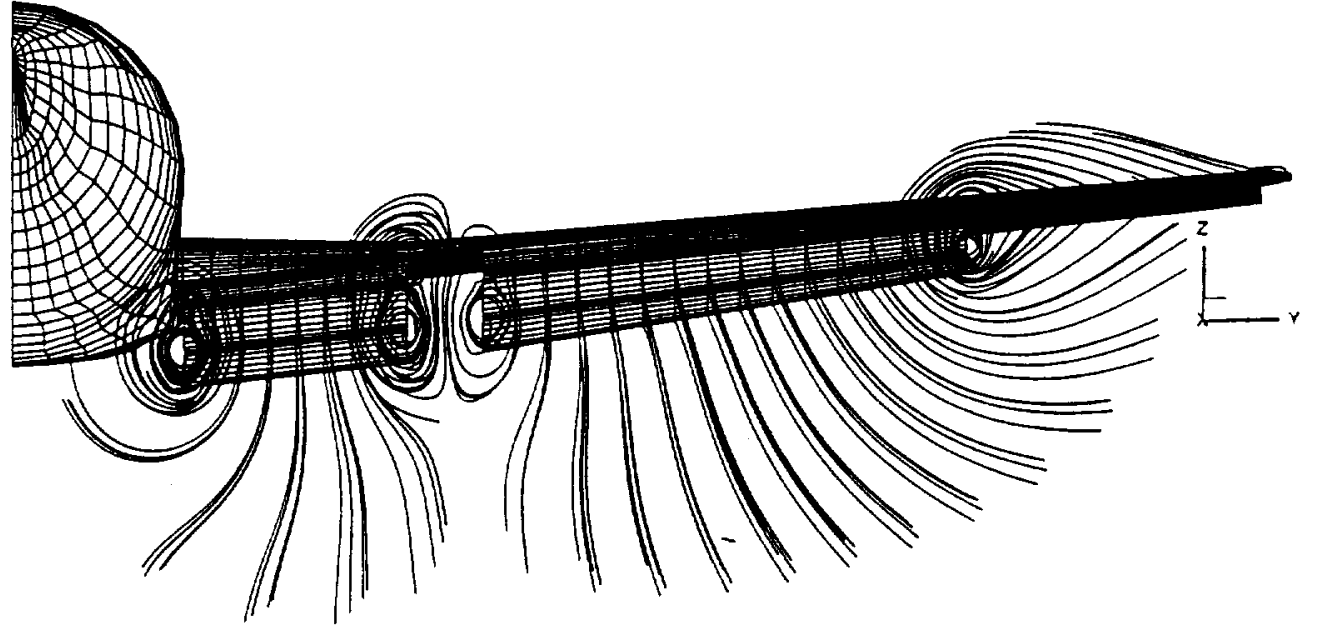

Figure 4. Off-body Streamlines Traces $\left(\alpha=7.6^{\circ}\right)$ 


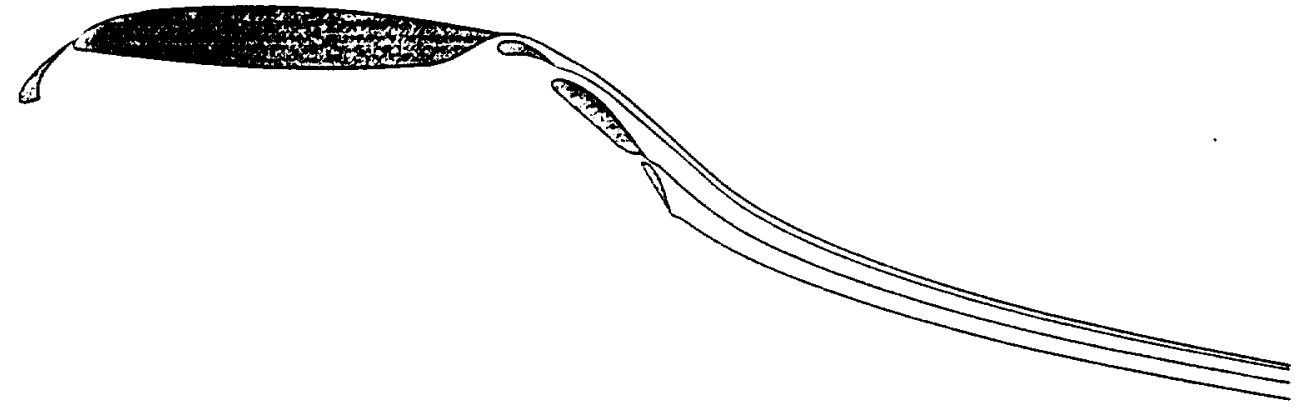

Figure 5. Cross-section of Streamlines at $\eta=0.58$

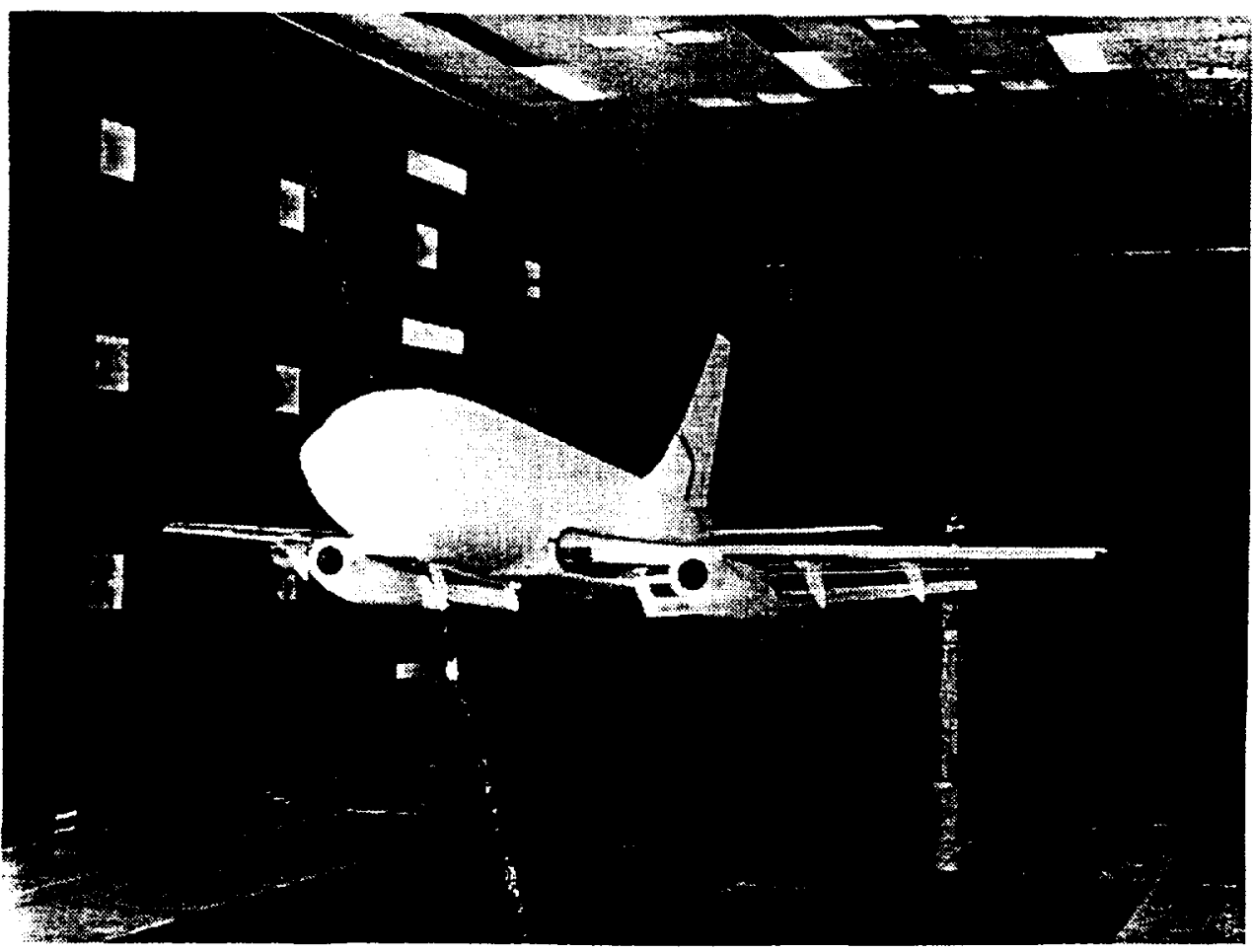

Figure 6. 1/8 Scale $737-100$ Model Installed in Langley's $14 \times 22$ Wind Tunnel 


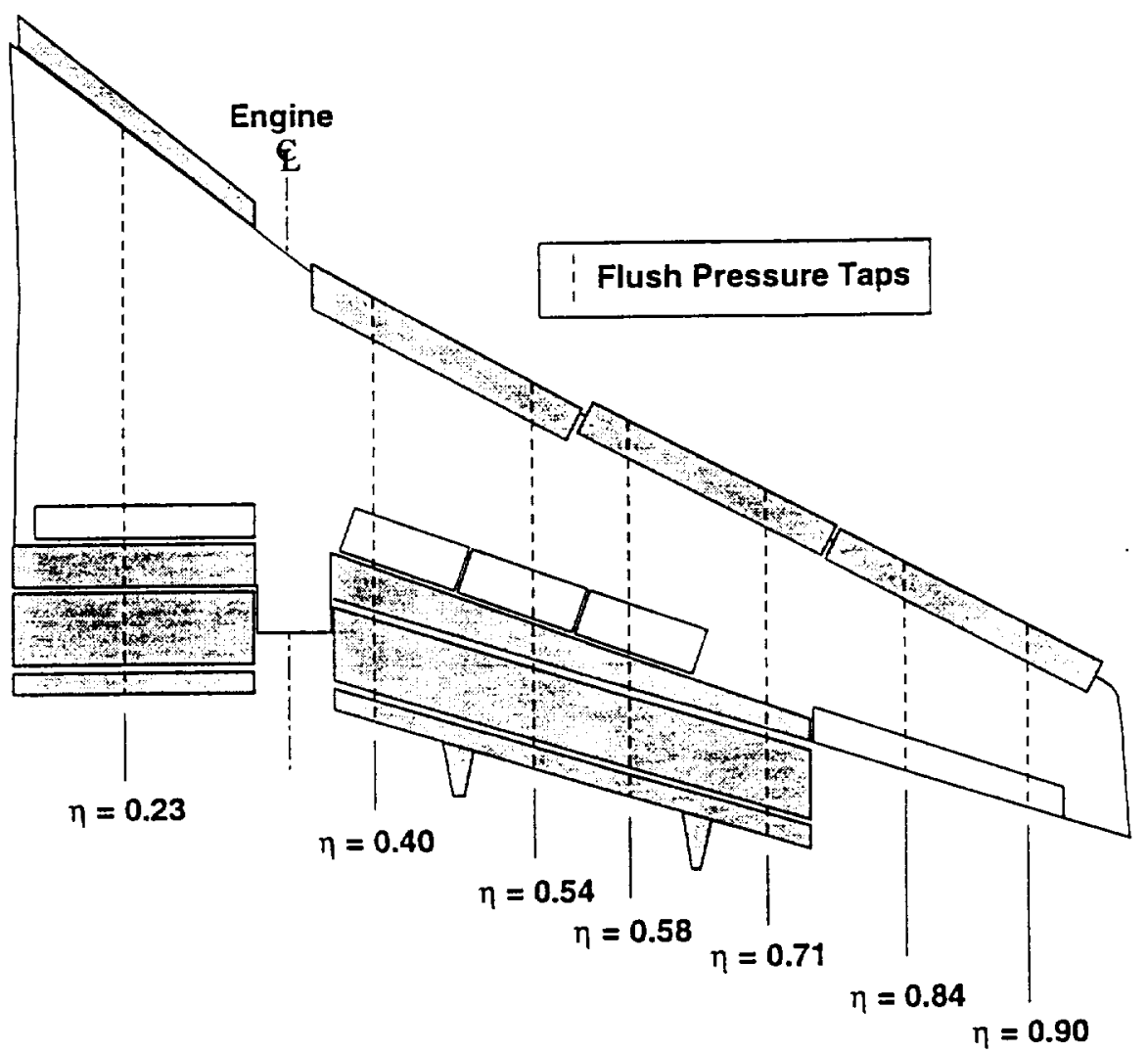

Figure 7. Spanwise Pressure Tap Locations on $1 / 8$ Scale Model

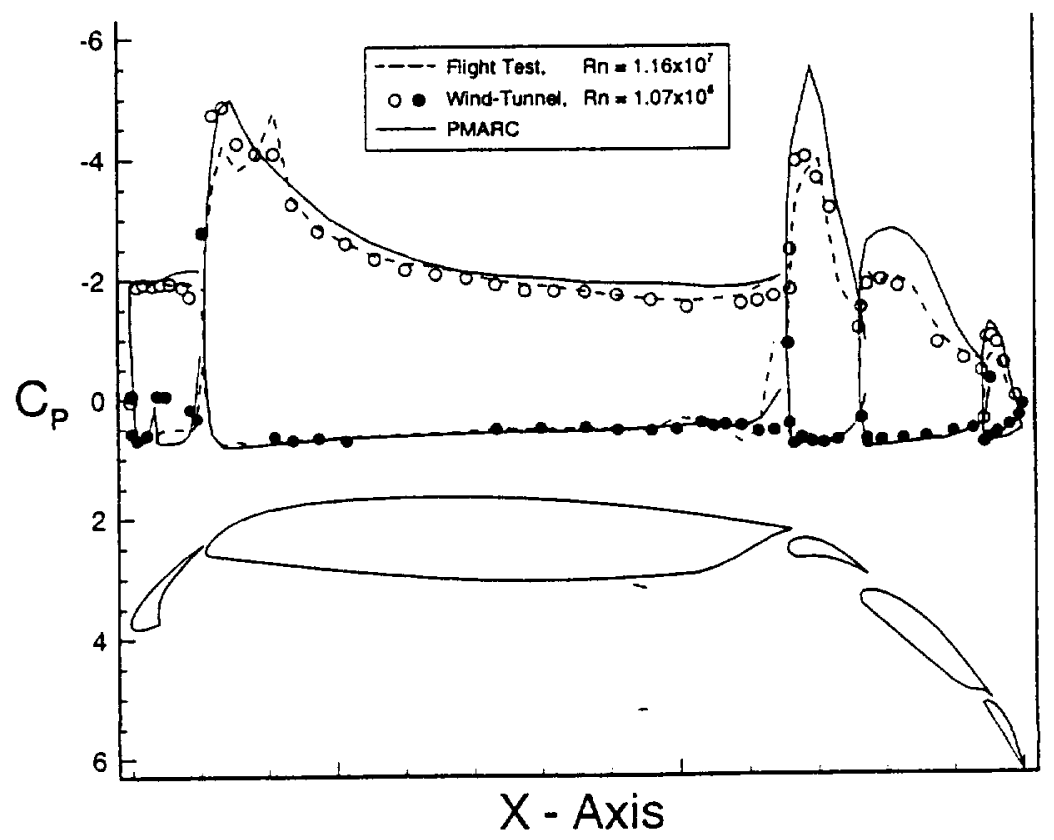

Figure 8. $C_{P}$ Comparisons: Wind-Tunnel, PMARC and Flight $\left(\alpha=8.0^{\circ}, \eta=0.58\right)$ 


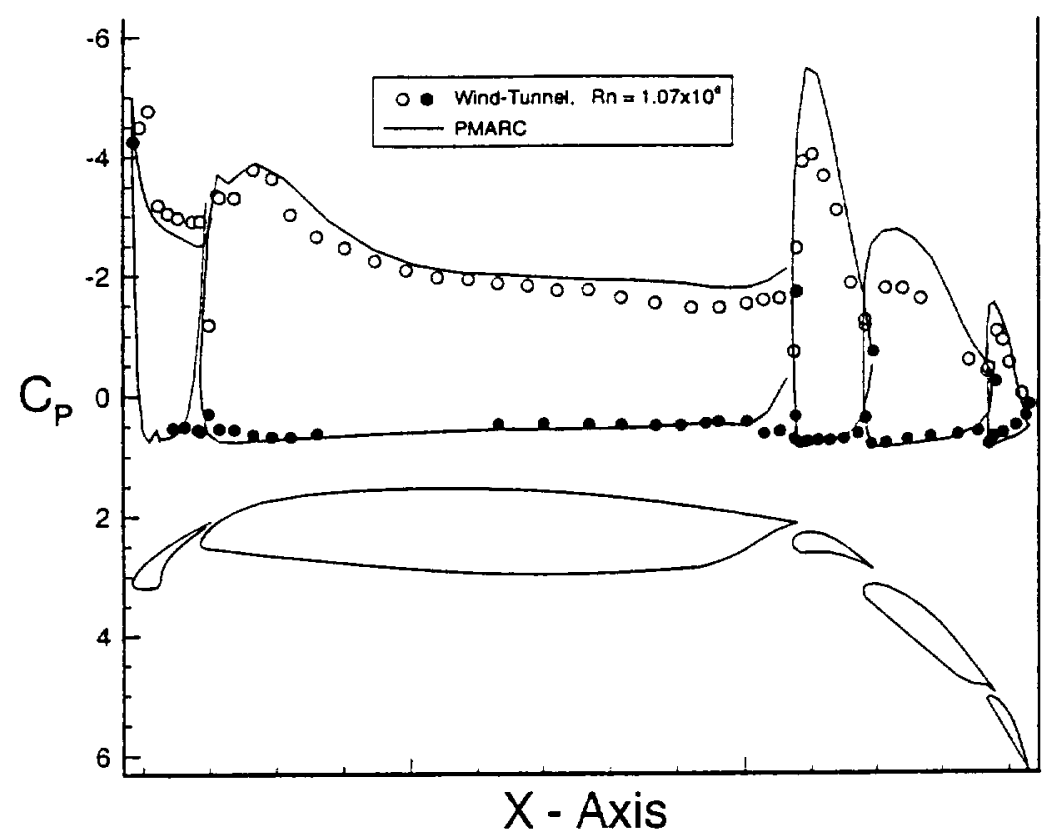

Figure 9. $C_{P}$ Comparisons: Wind-Tunnel and PMARC $\left(\alpha=8.0^{\circ}, \eta=0.54\right)$

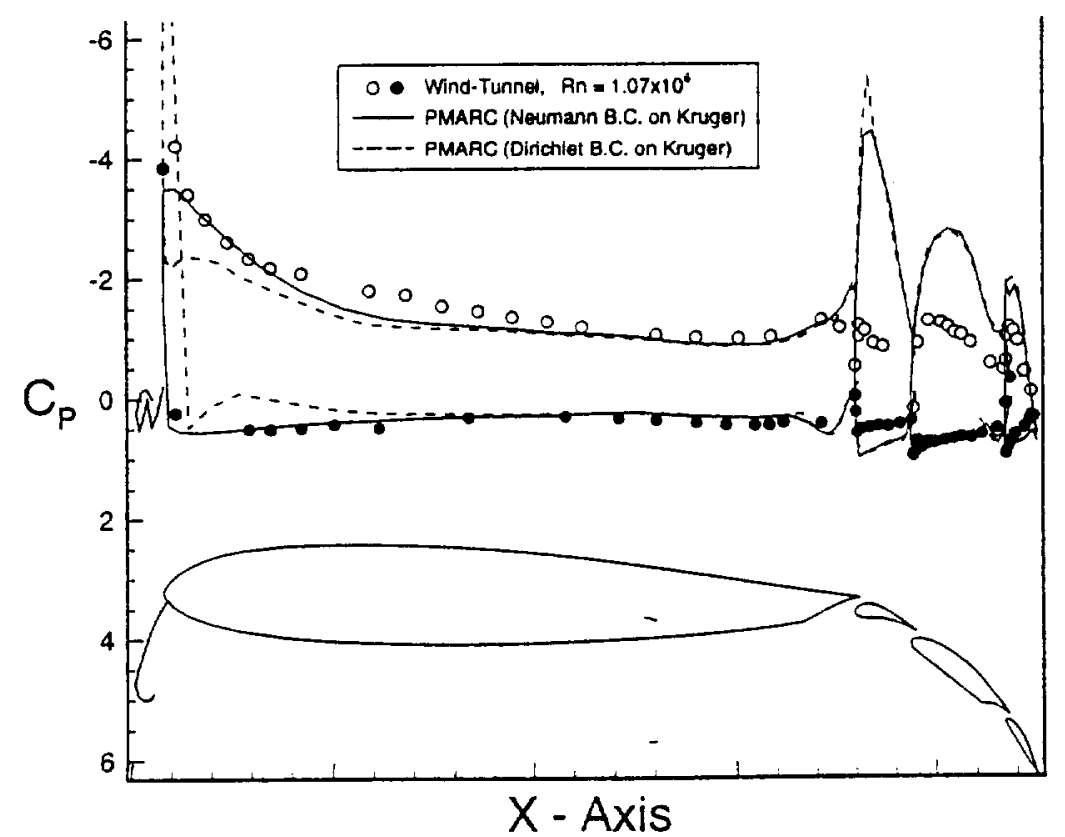

Figure 10. $C_{P}$ Comparisons: Wind-Tunnel and PMARC $\left(\alpha=8.0^{\circ}, \eta=0.23\right)$ 


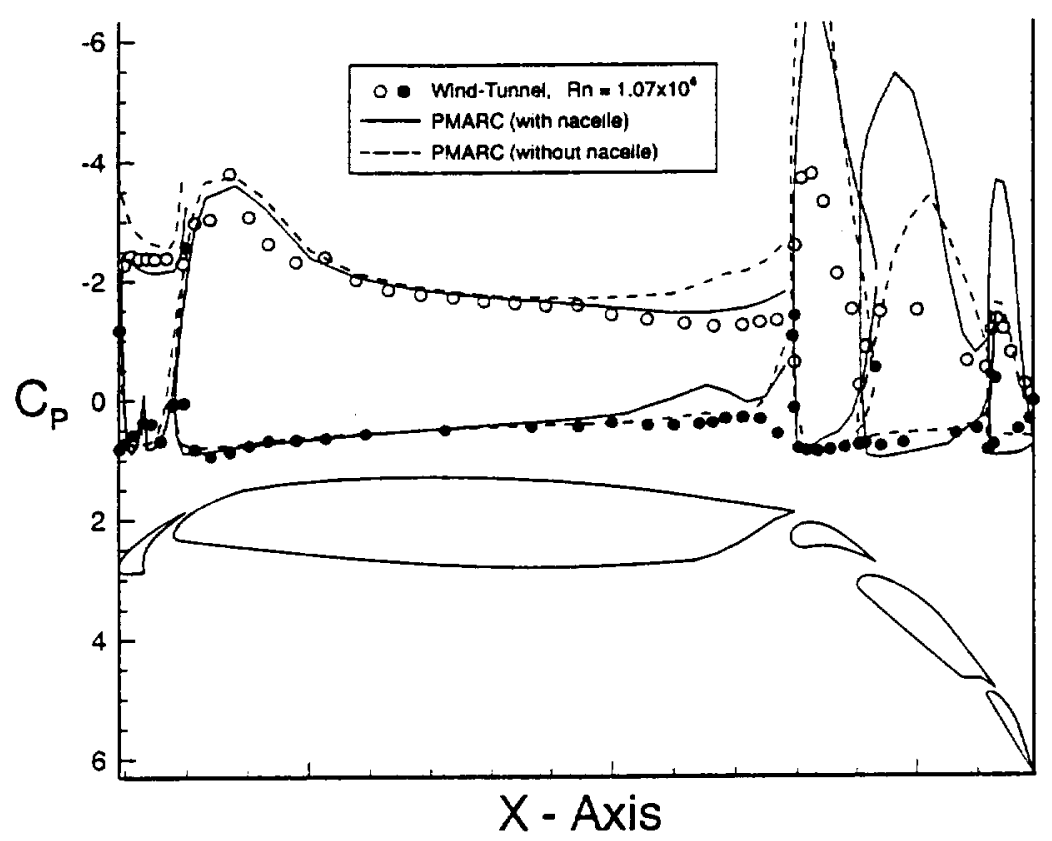

Figure 11. $C_{P}$ Comparisons: Wind-Tunnel and PMARC $\left(\alpha=8.0^{\circ}, \eta=0.40\right)$

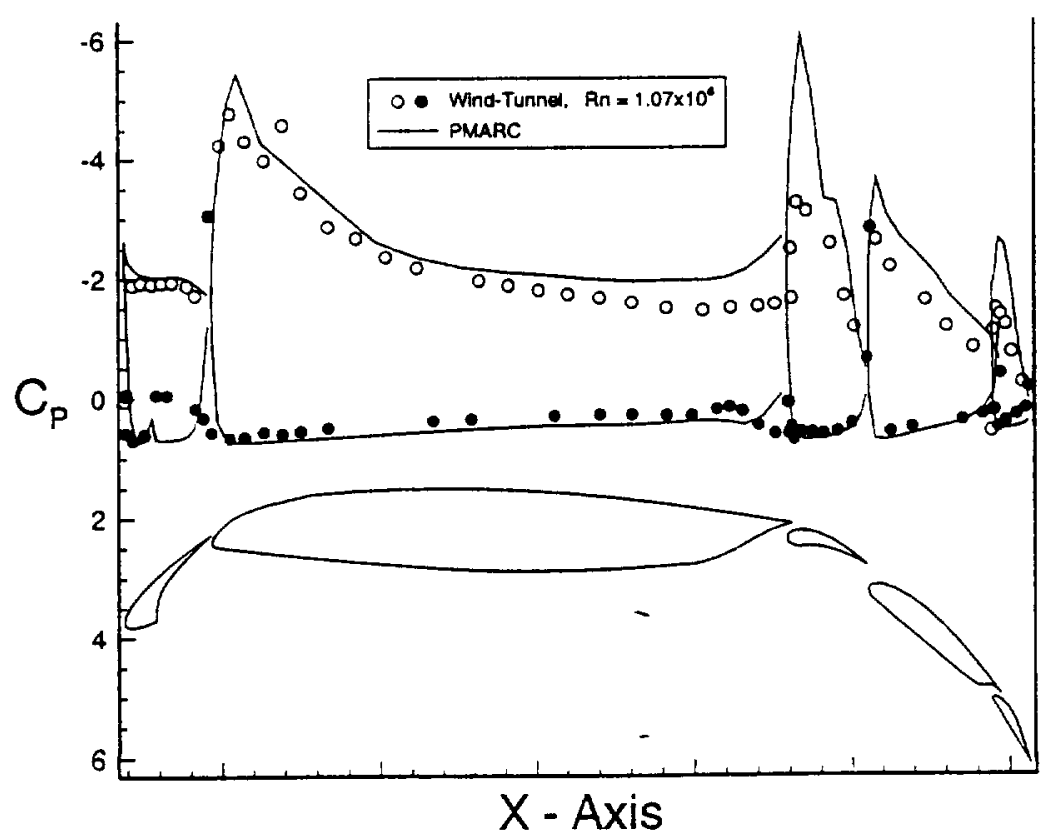

Figure 12. $C_{P}$ Comparisons: Wind-Tunnel and PMARC $\left(\alpha=8.0^{\circ}, \eta=0.71\right)$ 